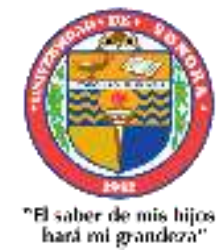

( Julio - Diciembre 2020)

Año 13.

Revista de Investigación

Núm. 33

Académica sin Frontera

ISSN: 2007-8870

https://revistainvestigacionacademicasinfrontera.unison.mx/index.php/RDIASF

Recibido el 27 de junio de 2020. Dictaminado mediante arbitraje favorablemente 7 de octubre de 2020 .

\title{
CONSTRUYENDO UN ECOSISTEMA EMPRENDEDOR
}

\author{
Guadalupe Zuberl Ochoa Cabrera
}

Ricardo Arturo Valenzuela Ramos

\section{Correos: zubeoc@hotmail.com y ricardovzla@outlook.com}

\section{Resumen}

Hablar de emprendimiento es hacer, es crear y es impulsar aquella convicción que generará oportunidades de crecimiento y desarrollo, ya sea social o empresarial. Conocer las herramientas y alternativas que ofrece el emprendedurismo es valorar las posibilidades de crecer y consolidarse. Comunidades como Silicon Valley, Boston, Berlín, Tel Aviv, Londres y Boulder tardaron décadas en convertirse en ecosistemas de innovación y emprendimiento avanzados y sólidos. Sin embargo, las grandes ideas de negocios pueden venir y surgir de cualquier parte hoy en día. En la economía moderna, estos ecosistemas pueden crecer en cualquier lugar, cada comunidad tiene la oportunidad de convertirse en un ecosistema próspero. De hecho, muchos ya lo han hecho. La creación de estos ecosistemas está ganando impulso en muchas partes del mundo ya no sólo en Estados Unidos a medida que se reconocen sus beneficios; influyendo también en México quien está empezando a adoptar este mecanismo. Estos ecosistemas ayudan a impulsar la innovación, la creación de nuevas empresas, a lograr cambios sociales y ayudan a que las oportunidades de empleo crezcan. Naciones, ciudades, regiones, universidades y otros están colaborando para establecer ecosistemas de emprendimiento como un componente crítico. El concepto de ecosistema se toma de la biología, donde se hace referencia a una comunidad de seres vivos y el medio natural en que viven. Donde los procesos vitales se relacionan entre sí y se desarrollan en función de los factores físicos de un mismo ambiente. Respecto al emprendimiento, el concepto se adapta para referirse a un medio que facilite a las Startups, PyMEs y prácticamente cualquier tipo de empresa desarrollarse y crecer a sus máximas capacidades. Un medio donde existan o se creen políticas públicas acordes, acceso a diferentes fuentes de financiamiento, capacitación y educación continua, inversión privada y vinculación con los diferentes sectores económicos como cámaras y organizaciones de la sociedad civil y universidades. La esencia de un ecosistema emprendedor es su gente. La cultura de confianza y colaboración que se desarrolla permite interactuar con éxito. Este tipo de ecosistemas permite el flujo rápido de talento, información y recursos. Ayuda a los emprendedores a encontrar rápidamente lo que necesitan en cada etapa de crecimiento. Como resultado, el todo es mayor que la suma de sus partes separadas.

Palabras clave: Ecosistema, emprendimiento, PYMES 


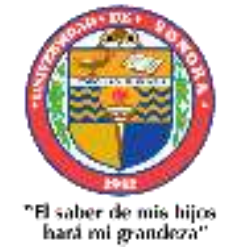

Año 13.

Núm. 33
( Julio - Diciembre 2020)

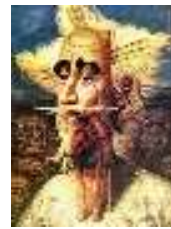

Revista de Investigación

Académica sin Frontera

ISSN: 2007-8870

https://revistainvestigacionacademicasinfrontera.unison.mx/index.php/RDIASF

Recibido el 27 de junio de 2020. Dictaminado mediante arbitraje favorablemente 7 de octubre de 2020 .

Introducción

Hablar de emprendimiento es hacer, es crear y es impulsar aquella convicción que generará oportunidades de crecimiento y desarrollo, ya sea social o empresarial. Conocer las herramientas y alternativas que ofrece el emprendedurismo es valorar las posibilidades de crecer y consolidarse.

Comunidades como Silicon Valley, Boston, Berlín, Tel Aviv, Londres y Boulder tardaron décadas en convertirse en ecosistemas de innovación y emprendimiento avanzados y sólidos. Sin embargo, las grandes ideas de negocios pueden venir y surgir de cualquier parte hoy en día. En la economía moderna, estos ecosistemas pueden crecer en cualquier lugar, cada comunidad tiene la oportunidad de convertirse en un ecosistema próspero. De hecho, muchos ya lo han hecho.

La creación de estos ecosistemas está ganando impulso en muchas partes del mundo ya no sólo en Estados Unidos a medida que se reconocen sus beneficios; influyendo también en México quien está empezando a adoptar este mecanismo. Estos ecosistemas ayudan a impulsar la innovación, la creación de nuevas empresas, a lograr cambios sociales y ayudan a que las oportunidades de empleo crezcan.

Naciones, ciudades, regiones, universidades y otros están colaborando para establecer ecosistemas de emprendimiento como un componente crítico.

El concepto de ecosistema se toma de la biología, donde se hace referencia a una comunidad de seres vivos y el medio natural en que viven. Donde los procesos vitales se relacionan entre sí y se desarrollan en función de los factores físicos de un mismo ambiente. Respecto al emprendimiento, el concepto se adapta para referirse a un medio que facilite a las Startups, PyMEs y prácticamente cualquier tipo de empresa desarrollarse y crecer a sus máximas capacidades. Un medio donde existan o se creen políticas públicas acordes, acceso a diferentes fuentes de financiamiento, capacitación y educación continua, inversión privada y vinculación con los diferentes sectores económicos como cámaras y organizaciones de la sociedad civil y universidades.

La esencia de un ecosistema emprendedor es su gente. La cultura de confianza y colaboración que se desarrolla permite interactuar con éxito. Este tipo de 
"Fl suber ide mis hijice

Año 13.

Núm. 33

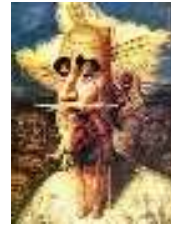

Revista de Investigación

Académica sin Frontera

ISSN: 2007-8870

https://revistainvestigacionacademicasinfrontera.unison.mx/index.php/RDIASF

Recibido el 27 de junio de 2020. Dictaminado mediante arbitraje favorablemente 7 de octubre de 2020 .

ecosistemas permite el flujo rápido de talento, información y recursos. Ayuda a los emprendedores a encontrar rápidamente lo que necesitan en cada etapa de crecimiento. Como resultado, el todo es mayor que la suma de sus partes separadas.

\section{El emprendimiento en México.}

Vale la pena preocuparse por el emprendimiento porque produce innovaciones que pueden hacer que los bienes y servicios sean más baratos y menos intensivos en recursos para todos. Los emprendedores también proporcionan empleo a otros en el proceso.

Según el Banco Mundial:

"La actividad emprendedora es un pilar del crecimiento económico. Para evidenciar el poder económico del emprendimiento, no necesitamos buscar más allá de los Estados Unidos, donde se ha demostrado que las empresas jóvenes son una fuente más importante de creación de empleo que las empresas establecidas ".

Según la OCDE:

"El emprendimiento y los emprendedores han sido reconocidos como fuentes importantes de innovación y, por lo tanto, también de crecimiento y empleo".

En el siguiente trabajo se abordará la importancia que representan en el desarrollo social y económico de una región los ecosistemas de emprendimiento. Antes de entrar de lleno en el tema, es necesario abordar el tema del desarrollo económico y ver el contexto mundial y local en sus principales índices.

México cayó seis posiciones en el ranking Doing Business 2020, el país se ubicó en la posición 60 luego de que en el reporte para 2019 se colocara en 54 . Doing Business mide la facilidad de hacer negocios en 190 países, para ello analiza 10 indicadores sobre regulaciones empresariales y la protección de los derechos de propiedad que miden los procedimientos, tiempos y costos federales y locales que impactan el ambiente de negocios. 
Año 13.

Núm. 33

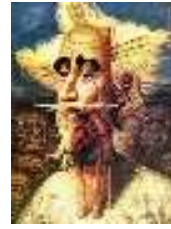

Revista de Investigación

Académica sin Frontera ISSN: 2007-8870

https://revistainvestigacionacademicasinfrontera.unison.mx/index.php/RDIASF

Recibido el 27 de junio de 2020. Dictaminado mediante arbitraje favorablemente 7 de octubre de 2020 .

\section{Los pilares en el país}

\begin{tabular}{lccllrr} 
& Lugar de 190 economias & & \multicolumn{2}{c}{ Lugar de 190 economias } \\
& $\mathbf{2 0 1 9}$ & $\mathbf{2 0 2 0}$ & & $\mathbf{2 0 1 9}$ & $\mathbf{2 0 2 0}$ \\
Apertura de negocio & 94 & 107 & Protección de los inversionistas minoritarios & 72 & 61 \\
Permisos de cosntrucción & 93 & 93 & Pago de impuestos & 116 & 120 \\
Registro de propiedades & 103 & 105 & Comercio transfronterizo & 66 & 69 \\
Obtención de electricidad & 99 & 106 & Cumplimiento de contratos & 43 & 43 \\
Obtención de crédito & 8 & 11 & Resolución dela lansolvencia & 32 & 33
\end{tabular}

Fuente: Banco Mundial

Tabla1: Comparación 2019-2020

Las regulaciones, que son analizadas por el Banco Mundial, afectan 12 áreas del ciclo de vida de una empresa. Diez de estas áreas están incluidas en el puntaje y la clasificación de este año: apertura de una empresa, manejo de permisos de construcción, obtención de electricidad, registro de propiedades, obtención de crédito, protección de los inversionistas minoritarios, pago de impuestos, comercio transfronterizo, cumplimiento de contratos y resolución de insolvencia.

Con la caída, el país también perdió el primer lugar de América Latina, ahora en manos de Chile, que se posicionó en el puesto 59. En la clasificación de la región le siguen: Puerto Rico (65), Colombia (67), Jamaica (71), Costa Rica (74), Perú (76), Panamá (86). Brasil se ubicó en el sitio 124 y Argentina quedó en el 126.

Nueva Zelanda obtuvo una calificación de 86.8 puntos, por lo que se colocó en la primera posición del reporte seguida por Singapur, Hong Kong, Dinamarca y Corea del Sur. Estados Unidos se ubica en el puesto número 
https://revistainvestigacionacademicasinfrontera.unison.mx/index.php/RDIASF

Recibido el 27 de junio de 2020. Dictaminado mediante arbitraje favorablemente 7 de octubre de 2020 .

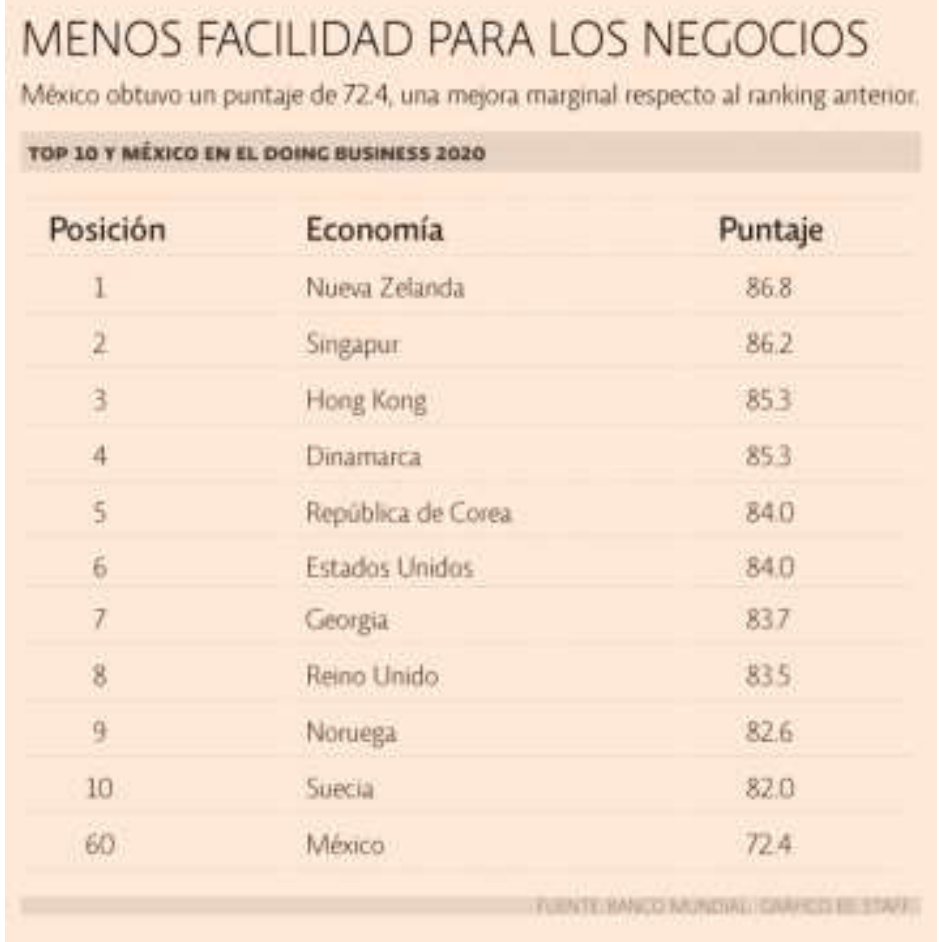

Tabla2: México Doing Business 2020.

Por otra parte, el Índice Global de Emprendimiento desarrollado por el Instituto de Emprendimiento Global y Desarrollo (GEDI, por sus siglas en inglés) es un índice anual que mide la salud de los ecosistemas de emprendimiento en cada uno de 137 países. Luego clasifica el desempeño de estos uno contra el otro. Esto proporciona una imagen de cómo se desempeña cada país en el contexto nacional e internacional.

La metodología GEDI recopila datos sobre las actitudes empresariales, las habilidades y las aspiraciones de la población local y luego las compara con la "infraestructura" social y económica predominante; esto incluye aspectos como la conectividad de banda ancha y los enlaces de transporte a mercados externos. Este proceso crea 14 "pilares" que GEDI utiliza para medir la salud del ecosistema regional. El primer lugar lo ocupa Estados Unidos, le siguen Suiza y Canadá. México ocupa el lugar 75. (The Global Entrepreneurship and Development Institute GEDI, 2018). 
Año 13.

Núm. 33

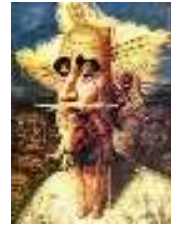

Revista de Investigación

Académica sin Frontera ISSN: 2007-8870

\section{https://revistainvestigacionacademicasinfrontera.unison.mx/index.php/RDIASF}

Recibido el 27 de junio de 2020. Dictaminado mediante arbitraje favorablemente 7 de octubre de 2020 .

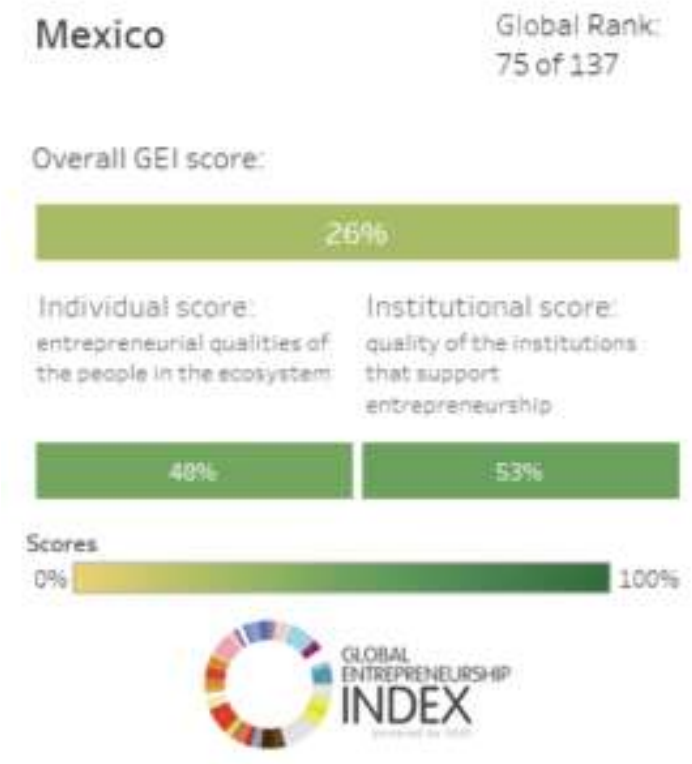

Strongest area

Networking
Weakest area Cultural Support
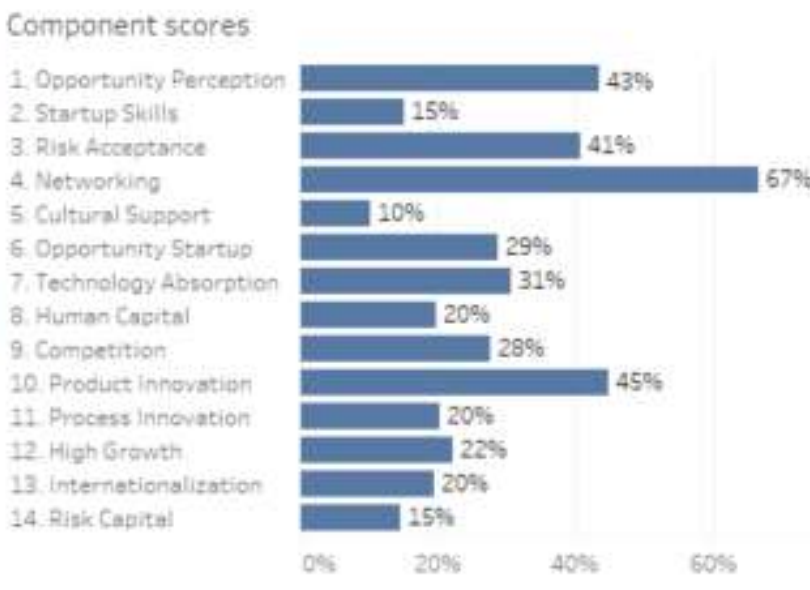

Tabla3: GEDI 2018 México.

El Índice de Desarrollo Humano (IDH), que mide la longevidad, educación e ingreso de la población, para el año 2017, sitúa a México en el lugar 74, dentro del grupo de países con nivel de desarrollo humano alto; con una expectativa de vida de 77,3 años, promedio de años de escolarización de 8,6 y un ingreso nacional bruto per cápita de US\$16,944 para 2017. Si se compara con Estados Unidos, se tiene que este país ocupa el lugar número 13 entre los 189 países que conforman este índice, tiene una expectativa de vida de vida de 79,5 años, promedio de años de escolarización de 13,4 y un ingreso nacional bruto per cápita de US\$ 54,941.

Resulta entonces necesario poder conocer la función e importancia que tiene el desarrollar ecosistemas emprendedores para cambiar y mejorar el desarrollo social y económico en ciudades o regiones que cuentan con condiciones más complicadas 0 adversas.

Ecosistemas de Emprendimiento. 


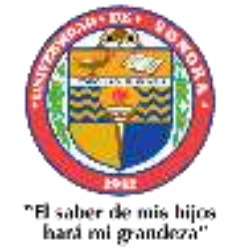

Año 13.

Núm. 33
( Julio - Diciembre 2020)

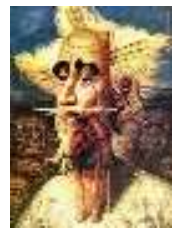

Revista de Investigación

Académica sin Frontera

ISSN: 2007-8870

https://revistainvestigacionacademicasinfrontera.unison.mx/index.php/RDIASF

Recibido el 27 de junio de 2020. Dictaminado mediante arbitraje favorablemente 7 de octubre de 2020 .

Hoy en día casi todo el mundo habla de "ecosistemas de emprendimiento" o "ecosistemas de start-ups". ¿Pero que son? En el lenguaje profesional, los ecosistemas emprendedores pueden significar cualquier cosa. Esto significa que su valor para guiar la acción política fácilmente equivale a nada.

Ante los grandes retos tanto sociales, económicos, empresariales y políticos a los que se enfrenta el mundo y en especial América Latina, en los últimos años se han buscado distintos modelos tanto públicos como privados para poder crear soluciones sostenibles que permitan reducir estas problemáticas, uno de estos modelos de trabajo son los ecosistemas de emprendimiento, que abarcan desde lo tecnológico, empresarial y social.

El concepto de ecosistema se toma de la biología, donde se hace referencia a una comunidad de seres vivos y el medio natural en que viven. Donde los procesos vitales se relacionan entre sí y se desarrollan en función de los factores físicos de un mismo ambiente. Respecto al emprendimiento, el concepto se adapta para referirse a un medio que facilite a las Startups, PyMEs y prácticamente cualquier tipo de empresa desarrollarse y crecer a sus máximas capacidades. Un medio donde existan o se creen políticas públicas acordes, acceso a diferentes fuentes de financiamiento, capacitación y educación continua, inversión privada y vinculación con los diferentes sectores económicos como cámaras y organizaciones de la sociedad civil y universidades.

El ecosistema emprendedor, como una idea propuesta por Daniel Isenberg en 2010, a menudo se entiende como un sistema adaptativo complejo, una constelación de conexiones entre componentes del ecosistema donde las relaciones entre elementos individuales son no lineales y dinámicas. Por lo tanto, el ecosistema es una estructura dinámica formada por organizaciones interconectadas e interdependientes que podrían ser las nuevas empresas, grandes corporaciones, gobiernos, asociaciones industriales, inversores individuales, organizaciones del sector público, universidades y centros de investigación. El sistema es complejo ya que las interacciones a nivel macro y micro nivel entre elementos individuales se influyen entre sí.

Comunidades como Silicon Valley, Boston, Berlín, Tel Aviv, Londres y Boulder tardaron décadas en convertirse en ecosistemas de innovación y emprendimiento 


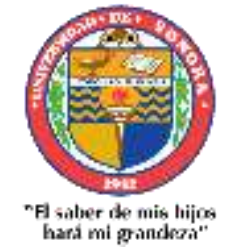

Año 13.

Núm. 33
( Julio - Diciembre 2020)

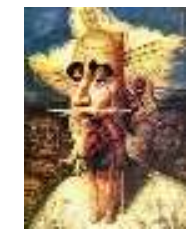

Revista de Investigación

Académica sin Frontera

ISSN: 2007-8870

https://revistainvestigacionacademicasinfrontera.unison.mx/index.php/RDIASF

Recibido el 27 de junio de 2020. Dictaminado mediante arbitraje favorablemente 7 de octubre de 2020 .

avanzados y sólidos. Sin embargo, las grandes ideas de negocios pueden venir y surgir de cualquier parte hoy en día. En la economía moderna, estos ecosistemas pueden crecer en cualquier lugar, cada comunidad tiene la oportunidad de convertirse en un ecosistema próspero. De hecho, muchos ya lo han hecho.

La creación de estos ecosistemas está ganando impulso en muchas partes del mundo ya no sólo en Estados Unidos a medida que se reconocen sus beneficios; influyendo también en México quien está empezando a adoptar este mecanismo. Estos ecosistemas ayudan a impulsar la innovación, la creación de nuevas empresas, a lograr cambios sociales y ayudan a que las oportunidades de empleo crezcan.

Los ecosistemas de emprendimiento están en plena efervescencia. Las diferentes crisis, los cambios en los sectores tradicionales financieros, la volatilidad de la bolsa, las pocas oportunidades de empleo, la tecnología, los servicios en la nube, los cambios de hábito de los consumidores, la creación de nuevos modelos de negocio, son solo una muestra de lo que ha propiciado el nacimiento de diferentes ecosistemas emprendedores.

Para algunos, solo es una enorme burbuja como lo sucedido con las punto com y para otros, una actividad fundamental que todavía tiene que desarrollarse, madurar y sobre todo profesionalizarse.

Para que un ecosistema emprendedor funcione, hacen falta tres pilares básicos: emprendedores, educación e inversión. La proporción idónea de calidad y cantidad en cada uno de ellos es la clave para la creación de grandes hubs de emprendimiento a nivel mundial.

\section{Desarrollo de Ecosistemas de Emprendimiento.}

El concepto de ecosistemas de emprendimiento ha surgido en los últimos años como un marco para comprender la naturaleza de los lugares en los que florece la actividad emprendedora. Spigel (2017) los define como: 'combinaciones de elementos sociales, políticos, económicos y culturales dentro de una región que apoyan el desarrollo y el crecimiento de nuevas empresas innovadoras y alientan a 


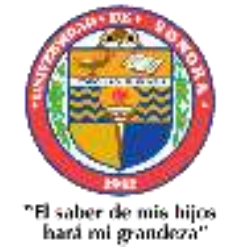

Año 13.

Núm. 33
( Julio - Diciembre 2020)

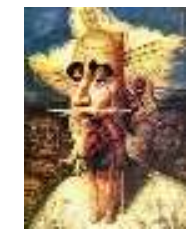

Revista de Investigación

Académica sin Frontera

ISSN: 2007-8870

https://revistainvestigacionacademicasinfrontera.unison.mx/index.php/RDIASF

Recibido el 27 de junio de 2020. Dictaminado mediante arbitraje favorablemente 7 de octubre de 2020 .

emprendedores nacientes y otros actores a correr el riesgo de comenzar, financiación $\mathrm{y}$, de otro modo, asistencia a empresas de alto riesgo.

Para el desarrollo de los ecosistemas de emprendimiento, es necesario considerar varios factores a cambiar como pasar el enfoque donde solo incuban negocios a incubar personas. Para que de esta manera se puedan desarrollar como individuos. A medida que los temas de automatización, robotización e inteligencia artificial complican el panorama futuro de los empleos y donde en este futuro se requiere personas con "habilidades blandas" mejor desarrolladas, se vuelve un punto muy importante el incubar personas.

Una vez que hay individuos desarrollados es entonces cuando se vuelve también parte importante el incubar comunidades. Fomentar la colaboración entre las comunidades de emprendedores debe volverse una acción constante que permita la creación de redes de emprendedores. Es importante destacar que la creación o incubación de comunidades no se debe limitar solamente a la creación de redes de personas o alianzas estratégicas. se debe de ir más allá y fomentar la creación de espacios donde se pueda dar acompañamiento entre emprendedores.

El hecho de que a través de estos espacios los diferentes emprendedores puedan convivir con pares en distintas industrias e incluso países, genera aprendizajes, relaciones interpersonales y redes de soporte que, aunque intangibles, son clave para el éxito de los emprendedores.

Los atributos de un ecosistema no existen de forma aislada, sino que se desarrollan en conjunto, lo que ayuda a influirse y reproducirse entre sí. Por ejemplo, las creencias subyacentes de una comunidad sobre el estatus social más amplio del emprendimiento afectan el deseo de los actores emprendedores de apoyar los esfuerzos emprendedores de otros. Al normalizar y legitimar el apoyo al emprendimiento dentro de la comunidad en general, los atributos culturales de un ecosistema crean un contexto a través del cual pueden surgir atributos sociales de apoyo. Esto contribuye a la formación de redes densas entre emprendedores, inversores y asesores.

Políticas para ecosistemas de emprendimiento. 


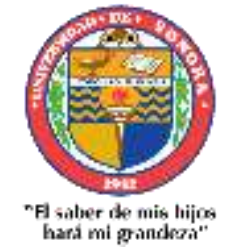

Año 13.

Núm. 33
( Julio - Diciembre 2020)

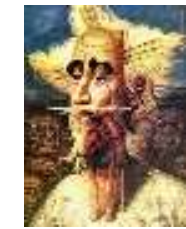

Revista de Investigación

Académica sin Frontera

ISSN: 2007-8870

https://revistainvestigacionacademicasinfrontera.unison.mx/index.php/RDIASF

Recibido el 27 de junio de 2020. Dictaminado mediante arbitraje favorablemente 7 de octubre de 2020 .

Facilitar el emprendimiento es una de las principales prioridades de las políticas gubernamentales. Las políticas de apoyo al emprendimiento se han vuelto cada vez más sofisticadas con el tiempo, a medida que los gobiernos han pasado de facilitar la creación de nuevas empresas en general a tratar de apoyar a las empresas de alto crecimiento. Actualmente, muchos gobiernos hablan de "ecosistemas de apoyo" o "ecosistemas de soporte" que cubren todo el ciclo de vida de la nueva empresa, desde el inicio hasta la supervivencia temprana, el crecimiento temprano y la expansión internacional. Sin embargo, si bien las carteras de políticas están creciendo, la mayoría de las políticas aún adolecen de una falta de comprensión general de lo que impulsa y limita el emprendimiento productivo en una economía determinada. Aunque los encargados de formular políticas hablan cada vez más de "ecosistemas de emprendimiento", las políticas de emprendimiento realmente no consideran ni abordan los cuellos de botella que frenan el rendimiento del sistema. Como resultado, las carteras de políticas de emprendimiento siguen sin poder cambiar realmente cómo funciona el ecosistema.

La falta de investigación académica y teoría no ayuda. Los investigadores de emprendimiento han invertido poco esfuerzo en definir qué significan realmente los "ecosistemas de emprendimiento" y cómo funcionan. Como resultado, cuando los gobiernos y los investigadores hablan de "ecosistemas de emprendimiento", pueden significar cosas completamente diferentes. Esta confusión socava el diseño y la implementación de políticas que apoyan efectivamente los tipos de actividad empresarial que pueden marcar una diferencia real para el crecimiento económico.

Con el fin de diseñar políticas efectivas para mejorar los ecosistemas de emprendimiento, los encargados de formular políticas deben seguir un enfoque de ecosistemas verdadero y no simplemente hablar de ello. Es importante destacar que los encargados de elaborar políticas deben darse cuenta de que, a nivel de país, el emprendimiento realmente se entiende mejor como un sistema y no simplemente como una mera suma de esfuerzos individuales. Los legisladores no pueden simplemente contar el número de emprendedores en la economía y pensar que cuantos más emprendedores haya, mayor será la contribución que estos pueden hacer al crecimiento económico. No todos los emprendedores son iguales: solo unos pocos emprendedores nuevos hacen crecer su negocio para generar niveles significativos de empleo y alcanzar niveles significativos de productividad económica. 


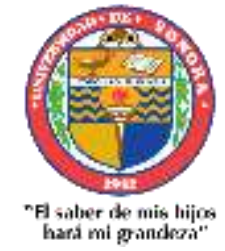

Año 13.

Núm. 33
( Julio - Diciembre 2020)

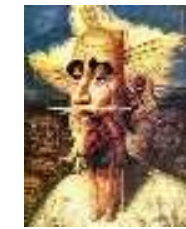

Revista de Investigación

Académica sin Frontera

ISSN: 2007-8870

https://revistainvestigacionacademicasinfrontera.unison.mx/index.php/RDIASF

Recibido el 27 de junio de 2020. Dictaminado mediante arbitraje favorablemente 7 de octubre de 2020 .

Por lo tanto, las políticas que no consideran la calidad de la actividad empresarial probablemente no sean efectivas.

En segundo lugar, no todas las economías son iguales. Diferentes economías tienen diferentes fortalezas y desafíos, y sus ecosistemas de emprendimiento pueden sufrir diferentes cuellos de botella. Por ejemplo, las políticas de financiamiento podrían no ser efectivas si el cuello de botella es la falta de aspiraciones o, por ejemplo, el fracaso de individuos bien educados para elegir el emprendimiento como una opción profesional. Una vez más, quién inicia nuevas empresas es una pregunta mucho más importante que cuántas personas lo hacen.

Tercero, el emprendimiento no se trata solo de individuos: también el contexto importa. Exactamente el mismo negocio tendrá perspectivas de crecimiento muy diferentes dependiendo de dónde se cree. Por ejemplo, la misma puesta en marcha de alta tecnología sería más probable que creara un impacto en Silicon Valley que, por ejemplo, algún país de bajos ingresos que carece de infraestructura para el emprendimiento productivo. El punto importante a entender es que el contexto económico y social del país, o sus condiciones para el emprendimiento, no solo influye en las perspectivas de la nueva empresa, sino también en quién inicia nuevas empresas en primer lugar. Es probable que las políticas que ignoran este aspecto de la dinámica empresarial no sean efectivas si las personas con las habilidades y actitudes adecuadas no pueden iniciar nuevas empresas.

Como demuestran los ejemplos anteriores, los ecosistemas de emprendimiento son complejos. Las políticas de emprendimiento deben reconocer esta complejidad y abordar el diseño de políticas en consecuencia. Debido a que los ecosistemas de emprendimiento son complejos y consisten en muchos elementos que interactúan, pueden ser muy difíciles de cambiar. Cuanto mayor es la complejidad del sistema, mayor es su inercia. Debido a esta inercia, las iniciativas políticas descoordinadas probablemente no creen un cambio duradero en la dinámica del ecosistema. Si las políticas no reconocen ni abordan los cuellos de botella que frenan el rendimiento del ecosistema, es probable que su efecto no sea duradero. Es probable que solo las políticas coordinadas de emprendimiento que aborden los cuellos de botella reales del ecosistema produzcan un cambio duradero en la dinámica del ecosistema. 


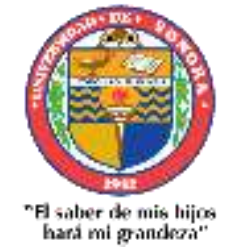

Año 13.

Núm. 33
( Julio - Diciembre 2020)

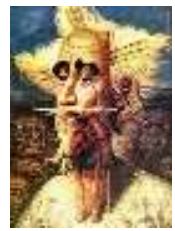

Revista de Investigación

Académica sin Frontera

ISSN: 2007-8870

https://revistainvestigacionacademicasinfrontera.unison.mx/index.php/RDIASF

Recibido el 27 de junio de 2020. Dictaminado mediante arbitraje favorablemente 7 de octubre de 2020 .

Políticas para ecosistemas de emprendimiento: tres desafíos clave.

Muchos gobiernos hablan de ecosistemas de emprendimiento. Si bien esto es excelente, pocos se han detenido a pensar en las implicaciones políticas de este fenómeno. ¿Cómo se diseñan políticas para facilitar los ecosistemas de emprendimiento? Para comprender este desafío, primero debemos entender cómo funciona el diseño de políticas.

La noción de falla del mercado está en el centro del diseño moderno de políticas: el gobierno interviene cuando el mercado no puede crear alguna actividad deseable por sí mismo. El ejemplo clásico es la inversión en I + D. La I + D es arriesgada: es difícil saber de antemano si el proyecto tendrá éxito. Por lo tanto, uno está tentado a dejar que otros corran el riesgo y luego simplemente "copiar" a estos proyectos exitosos. Sin embargo, si todos piensan de esta manera, nadie invertirá en I + D. Esta es la razón por la cual los gobiernos ofrecen todo tipo de subsidios para disminuir el riesgo de las empresas individuales.

Los subsidios de I + D abordan una falla de mercado específica y bien definida. Sin embargo, está mucho menos claro cuál es la falla del mercado que los ecosistemas de emprendimiento corrigen. En otras palabras, ¿cuál es el resultado deseable que crean los ecosistemas de emprendimiento? Muchos pensaran que la respuesta es más emprendedores y, por lo tanto, subsidiar la creación de nuevas empresas. Pero más emprendimiento no significa necesariamente un mejor emprendimiento, si las nuevas empresas no logran innovar y crecer. Posteriormente, los gobiernos descubrieron empresarios de alto crecimiento y pensaron que este era el secreto. Pero apoyar a las empresas de alto crecimiento no es trivial. ¡Sabemos que los gobiernos son notoriamente malos para elegir ganadores!

Los emprendedores de alto crecimiento o rendimiento son solo un producto que crean los ecosistemas de emprendimiento. El gran "servicio" proporcionado por los ecosistemas de emprendimiento es más general: es la asignación de recursos para usos productivos. Los emprendedores buscan oportunidades. Los ya exitosos generarán nueva riqueza. Esto implica que algo se hace de manera más productiva que antes. El resultado neto de la rotación empresarial de alta calidad es una mayor productividad y, por lo tanto, el crecimiento económico. 


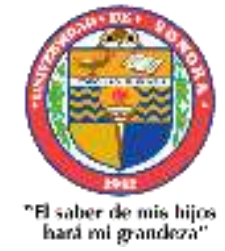

Año 13.

Núm. 33
( Julio - Diciembre 2020)

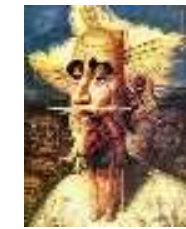

Revista de Investigación

Académica sin Frontera

ISSN: 2007-8870

https://revistainvestigacionacademicasinfrontera.unison.mx/index.php/RDIASF

Recibido el 27 de junio de 2020. Dictaminado mediante arbitraje favorablemente 7 de octubre de 2020 .

El aumento de la productividad en el emprendimiento es la característica definitiva de los ecosistemas de emprendimiento que funcionan bien. Pero este resultado, o la falta de él, no se remonta fácilmente a actividades específicas y fallas de mercado bien definidas. Esto es lo que diferencia el desafío político planteado por los ecosistemas de emprendimiento de la formulación de políticas más tradicionales. No podemos precisar fácilmente fallas de mercado específicas en los ecosistemas de emprendimiento, porque son muy complejas. Incorporan muchas partes interesadas diferentes que interactúan de muchas maneras diferentes. Es difícil para los legisladores (o cualquier otra persona) comprender cómo funciona exactamente el ecosistema y cuáles son las razones del bajo rendimiento. Esta complejidad explica por qué la mayoría de los intentos de copiar el fenómeno de Silicon Valley han fallado.

Se sugiere que la complejidad de los ecosistemas de emprendimiento crea tres desafíos claves para las políticas:

Comprender cómo funciona realmente el ecosistema de emprendimiento. Para diseñar políticas efectivas para los ecosistemas de emprendimiento, el primer desafío es comprender exactamente cómo funciona el ecosistema. Esto va más allá de simplemente describir la estructura estática del ecosistema. El verdadero desafío es descubrir cómo las partes interesadas del ecosistema interactúan e influyen entre sí para producir resultados del ecosistema, es decir, comprender qué impulsa la dinámica del ecosistema. Aquí, es fundamental reconocer y comprender los cuellos de botella que frenan el rendimiento del ecosistema.

Evitar consecuencias no deseadas. A veces las políticas son contraproducentes. Son contraproducentes exactamente porque es muy difícil comprender completamente los ecosistemas emprendedores complejos. Las intervenciones políticas mal diseñadas que no aborden los cuellos de botella reales del ecosistema pueden crear más daño que bien.

Superar la inercia del ecosistema. Otra consecuencia de la complejidad es que los ecosistemas de emprendimiento tienden a tener una alta inercia. Una vez que el ecosistema se establece en una operación más dada, es difícil empujarlo a otro modo, posiblemente mejor. Cambiar la dinámica del ecosistema es difícil, y las iniciativas de políticas aisladas corren el riesgo de no producir una diferencia perceptible en esta dinámica. Para superar la inercia del ecosistema, es importante identificar las 


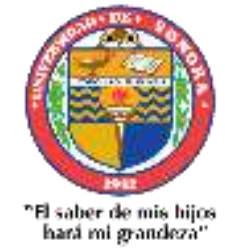

Año 13.

Núm. 33
( Julio - Diciembre 2020)

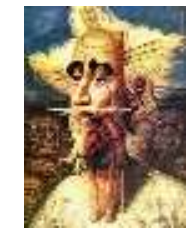

Revista de Investigación

Académica sin Frontera

ISSN: 2007-8870

https://revistainvestigacionacademicasinfrontera.unison.mx/index.php/RDIASF

Recibido el 27 de junio de 2020. Dictaminado mediante arbitraje favorablemente 7 de octubre de 2020 .

intervenciones políticas apropiadas para un ecosistema dado e implementarlas de manera coherente y bien orquestada. Solo las políticas bien orquestadas que aborden los cuellos de botella reales del ecosistema pueden esperar mejorar la dinámica del ecosistema.

Debido a estos tres desafíos, los datos "duros" que describen las entradas, salidas y recursos del ecosistema probablemente no sean suficientes para informar la política del ecosistema de emprendimiento. Por ejemplo, incluso con sus más de 30 variables, el Índice Global de Emprendimiento solo puede indicar dónde podrían estar al acecho los cuellos de botella del ecosistema. Para superar los tres desafíos, los datos "duros" deben complementarse con enfoques participativos que extraigan información "blanda" de los interesados en el ecosistema.

\section{Desarrollando una política efectiva para los ecosistemas de emprendimiento.}

La doctrina dominante en el análisis y diseño de políticas es apuntar y eliminar las fallas del mercado. Por ejemplo, los gobiernos otorgan préstamos blandos y otros fondos a los emprendedores para compensar la falla del mercado en proporcionar fondos para nuevos negocios. Debido a su falta de antecedentes, los nuevos emprendedores tienen dificultades para atraer fondos de instituciones financieras. Si el gobierno no interviniera, el mercado solo no lograría producir el resultado deseado, lo que inhibiría la creación y el crecimiento de nuevas empresas.

Este escenario es simple, engañosamente. Si la financiación es el verdadero cuello de botella, ¿por qué los capitalistas de riesgo a menudo se quejan de que la oferta de financiación de capital supera los proyectos invertibles? Si bien un aumento en los fondos blandos sin duda aumentaría la creación de nuevas empresas, ¿las nuevas empresas resultantes serían de alta calidad para ser invertibles? Y, ¿podría la financiación adicional crear resultados adversos, como la dependencia de los subsidios y el desplazamiento de la actividad de ángeles inversionistas?

Las consecuencias no deseadas y los resultados adversos pueden ser un desafío en cualquier dominio de políticas, pero particularmente en el espíritu emprendedor. Esto se debe a que el éxito de las nuevas empresas es incierto y depende de muchos factores. Para tener éxito, las empresas necesitan financiación, pero también equipos empresariales de alta calidad, servicios comerciales sólidos, el 


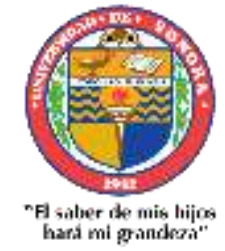

Año 13.

Núm. 33
( Julio - Diciembre 2020)

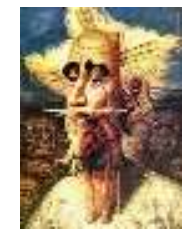

Revista de Investigación

Académica sin Frontera

ISSN: 2007-8870

https://revistainvestigacionacademicasinfrontera.unison.mx/index.php/RDIASF

Recibido el 27 de junio de 2020. Dictaminado mediante arbitraje favorablemente 7 de octubre de 2020 .

tipo adecuado de socios, empleados calificados, clientes receptivos y mucha suerte. Lo que puede parecer un fracaso directo del mercado en realidad puede ser un síntoma de algún problema más profundo que puede o no ser reparable con fondos blandos.

Las incertidumbres y complejidades de los ecosistemas de emprendimiento significan que los enfoques tradicionales de falla del mercado no son suficientes para las políticas de ecosistemas de emprendimiento. Los ecosistemas de emprendimiento son complejos: sus resultados se "producen" en innumerables interacciones entre muchos actores diferentes. Debido a esta complejidad, ningún individuo sabe realmente cómo funciona un ecosistema de emprendimiento específico. Sin embargo, el problema está en los detalles, ya que el éxito o el fracaso de las medidas de política individuales pueden depender de cuestiones relativamente menores en su aplicación. Debido a que muchos procesos en los ecosistemas de emprendimiento están interconectados, se pueden acumular cambios menores para crear un cambio importante no intencionado en la dinámica del ecosistema, aunque el resultado más habitual es que las medidas de política bien intencionadas, pero inadecuadamente dirigidas simplemente se disipan en el sistema sin ningún efecto perceptible.

¿Cuáles son las implicaciones de esto para el análisis y la implementación de políticas para los ecosistemas de emprendimiento? Si el diablo está en los detalles, la política necesita datos sobre los detalles. Pero la mayoría de las métricas disponibles para el análisis de políticas tienden a ser métricas de nivel macro: número de nuevas empresas creadas, cantidades de dinero invertidas, etc. Esto simplemente no es suficiente: necesita datos más detallados y mejores. Pero, ¿cómo se obtiene? Recuerda que, por definición, ningún individuo u organización puede tener una comprensión completa y completa de cómo funciona realmente el ecosistema.

Los enfoques de políticas para comprender y gestionar ecosistemas complejos se han estudiado en la literatura de "economía ecológica". Esta literatura explora las interacciones entre las sociedades humanas y los ecosistemas naturales que son similares a los ecosistemas de emprendimiento en complejidad.

- El análisis del ecosistema de emprendimiento requiere datos detallados. Los recuentos simples de empresas y las cifras de financiación de I + D no serán suficientes. Se necesitan datos detallados sobre muchos aspectos diferentes 


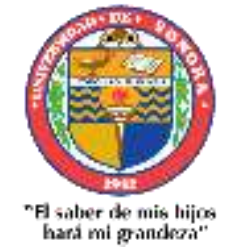

Año 13.

Núm. 33
Revista de Investigación

Académica sin Frontera

ISSN: 2007-8870

\section{https://revistainvestigacionacademicasinfrontera.unison.mx/index.php/RDIASF}

Recibido el 27 de junio de 2020. Dictaminado mediante arbitraje favorablemente 7 de octubre de 2020 .

de los procesos empresariales, como las actitudes, habilidades y aspiraciones empresariales. El índice GEI de ecosistemas de emprendimiento, por ejemplo, combina 15 variables que rastrean las actitudes, la capacidad y las aspiraciones empresariales de una muestra grande y representativa de individuos.

- Se necesitan datos a nivel individual y a nivel de sistema. Además de los datos de las personas, se necesitan datos sobre lo que se llama "condiciones del marco empresarial". Esto se debe a que la estructura y los recursos del ecosistema regulan la acción individual y sus resultados. El mismo individuo puede desempeñarse de manera diferente en diferentes contextos. El índice GEl combina datos sobre 15 "condiciones del marco empresarial" que regulan el desempeño de emprendedores individuales.

- Los datos "duros" deben combinarse con información "blanda" de las partes interesadas del ecosistema. No importa qué tan finos sean sus datos, no se puede obtener una comprensión completa de cómo funciona realmente el ecosistema. Para lograr esto, debe comprometerse con las partes interesadas del ecosistema para obtener sus perspectivas e ideas. Las ideas combinadas de múltiples partes interesadas revelarán perspectivas importantes e indocumentadas sobre el funcionamiento interno del ecosistema. Es por eso que el Proceso de Facilitación del Ecosistema de GEDI incluye numerosas reuniones de partes interesadas y discusiones de grupos focales.

En resumen, para diseñar una política efectiva para los ecosistemas de emprendimiento, se debe complementar los datos "duros" y detallados con enfoques participativos que involucren a los interesados del ecosistema. Solo los propios interesados en el ecosistema pueden proporcionar información "blanda" sobre el funcionamiento interno del ecosistema. Debido a que ninguna parte interesada individual tiene una visión completa, debe comprometerse con muchas partes interesadas diferentes, cada una con sus propias perspectivas para contribuir.

Sin embargo, extraer esa información "suave" no es simple. Se necesitan reuniones de las partes interesadas y discusiones de grupos focales de alcance más limitado, y cada discusión debe ser facilitada por una persona competente que sepa qué se está haciendo. El proceso de facilitación del ecosistema GEDI comienza con 


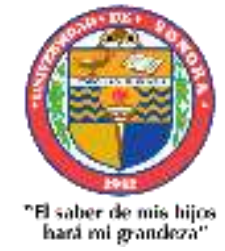

Año 13.

Núm. 33
( Julio - Diciembre 2020)

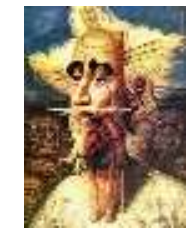

Revista de Investigación

Académica sin Frontera

ISSN: 2007-8870

https://revistainvestigacionacademicasinfrontera.unison.mx/index.php/RDIASF

Recibido el 27 de junio de 2020. Dictaminado mediante arbitraje favorablemente 7 de octubre de 2020 .

un debate general para llegar a un acuerdo sobre los cuellos de botella del ecosistema. El funcionamiento de cada cuello de botella se explora en discusiones de grupos focales. Una vez que se ha logrado una comprensión completa de los controladores de cuello de botella, comienza la búsqueda de soluciones.

Si se manejan y organizan cuidadosamente, los enfoques participativos pueden revelar una perspectiva coherente y basada en evidencia que no se puede lograr por otros medios.

El compromiso bien gestionado de las partes interesadas también ayuda a fomentar la conciencia mutua y el consenso entre las partes interesadas y aumentar el compromiso con las soluciones políticas acordadas. En el mejor de los casos, los enfoques participativos pueden desencadenar una acción política de abajo hacia arriba iniciada y coordinada por los propios interesados del ecosistema, eliminando así la necesidad de una acción de política "de arriba hacia abajo" por parte de los funcionarios. Pero el proceso debe gestionarse con habilidad, ya que las ideas pueden degenerar fácilmente en opiniones y consensos en conflictos. La investigación en economía ecológica sugiere que la participación de las partes interesadas mal implementada puede producir más daños que beneficios.

\section{Conclusión.}

Después de abordar la importancia y elementos a considerar para el desarrollo de los ecosistemas de emprendimiento, es interesante terminar esta serie de puntos con algunas conclusiones que permitan el análisis del por qué vale la pena implementar estos espacios.

A medida que la economía mundial continúa cada vez más complicada, los emprendedores y las "start-up's" están liderando el camino en la creación de nuevas innovaciones, nuevos productos, nuevos servicios y nuevos empleos. Están rejuveneciendo las economías de muchas ciudades de todo el mundo a medida que crean la base para la próxima ola de crecimiento económico, según el autor Brad Feld, empresario, inversor y mentor. Las nuevas empresas están transformando nuestra sociedad. En los últimos 100 años, pasamos de una era industrial, donde una estructura jerárquica dominaba los negocios y la sociedad, a una era posterior a la 
"Fl suler ide mis hijes

Año 13

Núm. 33

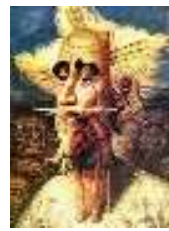

Revista de Investigación

Académica sin Frontera

ISSN: 2007-8870

https://revistainvestigacionacademicasinfrontera.unison.mx/index.php/RDIASF

Recibido el 27 de junio de 2020. Dictaminado mediante arbitraje favorablemente 7 de octubre de 2020 .

información en la que la red está interrumpiendo rápidamente la jerarquía y transformando la forma en que trabajamos y vivimos.

Un punto a tomar en cuenta es la detección de los "cuellos de botella". Cada región es diferente, por ende, cada ecosistema es diferente y representa diversos retos, para algunos son retos financieros mientras que para otros es a falta de inspiración o aspiración para emprender. Es importante detectar cuales son los retos con los que cuenta cada región.

Es importante recalcar que todas las partes que conforman un ecosistema juegan un papel crítico y relevante para crear las condiciones en el entorno para que se generen los emprendimientos de alto impacto y así mismo impulsarlos. Es necesario para la creación de un ecosistema de emprendimiento contar con políticas que permitan la intervención de los diferentes actores en principal la participación del gobierno y de los encargados de hacer políticas en nuestro país.

Por ejemplo, cuando el gobierno y sus entidades se involucran en el desarrollo de los ecosistemas de emprendimiento puede resultar alentador, porque están relacionados con el crecimiento económico y por lo tanto con la calidad de vida futura de las regiones. Las nuevas ideas de negocios, los emprendimientos y las nuevas empresas han demostrado jugar un papel fundamental en la innovación y su creación.

\section{Bibliografía}

Acs, Z.J., Autio, E., \& Szerb, L. (2014). National systems of entrepreneurship: Measurement issues and the process of knowledge creation. Progress in Human Geography, 28(1), 31-56.

Feld, B. (2012). Startup communities: Building an entrepreneurial ecosystem in your city. Hoboken, NJ: Wiley.

(2018). Global Entrepreneurship Index: The Global Entrepreneurship and Development Institute. https://thegedi.org/2018-global-entrepreneurship-index/.

(2019). Economy Profile Doing Business 2020 México: Doing Business. Recuperado de 
Revista de Investigación

Año 13.

Académica sin Frontera

Núm. 33

ISSN: 2007-8870

https://revistainvestigacionacademicasinfrontera.unison.mx/index.php/RDIASF

Recibido el 27 de junio de 2020. Dictaminado mediante arbitraje favorablemente 7 de octubre de 2020 .

https://www.doingbusiness.org/content/dam/doingBusiness/country/m/mexico/MEX.p df

Dr. Enrique Fernando Velázquez Contreras

Rector

Dr. Ramón Enrique Robles Zepeda

Secretario General Académico 
"Fl quber ile mis hijes

Año 13.

Núm. 33

https://revis
Revista de Investigación

Académica sin Frontera

ISSN: 2007-8870

Recibido el 27 de junio de 2020. Dictaminado mediante arbitraje favorablemente 7 de octubre de 2020 .

Dra. Rosa María Montesinos Cisneros

Secretaria General Administrativa

Dr. Rodolfo Basurto Álvarez

Director de Vinculación y Difusión

Dra. Adriana Leticia Navarro Verdugo

Vicerrectora de la Unidad Regional Sur

Dr. Ernesto Clark Valenzuela

Director de la División de Ciencias Económicas y Sociales

Dr. Francisco Espinoza Morales

Secretario de la División de Ciencias Económico y Sociales

Dra. Leticia María González Velásquez

Jefe del Departamento de Ciencias Económico Administrativas

Dra. Lidia Amalia Zallas Esquer

Jefe de Departamento de Ciencias Sociales

\title{
Comité Directivo
}

\author{
Editor Responsable
}

Dr. Francisco Espinoza Morales 
Año 13.

Núm. 33

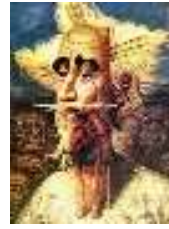

Revista de Investigación

Académica sin Frontera ISSN: 2007-8870

https://revistainvestigacionacademicasinfrontera.unison.mx/index.php/RDIASF

Recibido el 27 de junio de 2020. Dictaminado mediante arbitraje favorablemente 7 de octubre de 2020 .

\section{Directora}

Dra. Leticia María González Velásquez

\section{Subdirector}

Dr. Javier Carreón Guillen

\section{Editor Científico}

Dr. Cruz García Lirios

\section{Master Gráfico}

M.T.I. Francisco Alan Espinoza Zallas

Nos complace anunciar que su diario, "Academic Research Journal Withoutborders" (ISSN/EISSN 2007-8870) fue evaluado positivamente en la indexación Citefactor, ahora la página de la revista está disponible en línea, en caso de cualquier problema. Journals Master | International Innovative Journal Impact Factor (IIJIF)

Red Latinoamericana de revistas Académicas en Ciencias Sociales y Humanidades

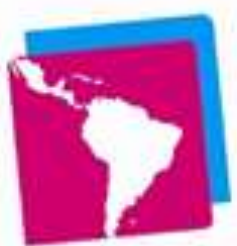

LatinREV

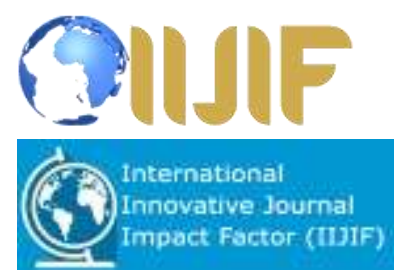

\section{Comité editorial}

Dra. Angélica María Rascón Larios

Universidad de Sonora. México

Dra. María del Rosario Molina González

Universidad de Sonora 
https://revistainvestigacionacademicasinfrontera.unison.mx/index.php/RDIASF

Recibido el 27 de junio de 2020. Dictaminado mediante arbitraje favorablemente 7 de octubre de 2020 .

Dra. Francisca Elena Rochin Wong

Universidad de Sonora. México

Dra. Lidia Amalia Zallas Esquer

Universidad de Sonora. México

Dra. Beatriz Llamas Arechiga

Universidad de Sonora. México

Dr. Rogelio Barba Álvarez

Universidad de Guadalajara. México

Dra. Rosa María Rincón Ornelas

Universidad de Sonora. México

Dr. Juan Flores Preciado

Universidad de Colima. México

Dr. Amado Olivares Leal. Universidad de Sonora

Universidad de Sonora. México

Dr. Guillermo Velázquez Valadez.

Instituto Politécnico Nacional (IPN) México

Dr. Hugo Nefstalí Padilla Torres.

Universidad Estatal de Sonora. México

Dr. Luis Ramón Moreno Moreno.

Universidad Autónoma de Baja California. México

Dr. Miguel Ángel Vázquez Ruiz.

Universidad de Sonora. México

Dra. Lorena Vélez García.

Universidad Autónoma de Baja California. México

Dra. Pabla Peralta Miranda.

Universidad Simón Bolívar, Barranquilla, Colombia

Mtro. Roberto Espíritu Olmos

Universidad de Colima (FCA Tecomán) Colima

Dr. Héctor Priego Huertas.

Universidad de Colima (FCA Tecomán) Colima

Mtra. María Guadalupe Alvarado Ibarra.

Universidad de Sonora. México. 
Recibido el 27 de junio de 2020. Dictaminado mediante arbitraje favorablemente 7 de octubre de 2020 .

MSc. Celso Germán Sánchez Zayas

Universidad de Camagüey, Ignacio Agramonte Loynaz, Cuba

Dra. María Luisa Quintero Soto

Universidad Autónoma del Estado de México

Dr. Eyder Bolivar Mojica

Universidad Católica, Luis Amigó, Medellin, Colombia

Revisores de Textos en Inglés

Mtro. Renato Encinas

Mtra. Cecilia Guadalupe Martínez Solano

\section{Comité científico}

Dr. Rosendo Martínez Jiménez. Universidad Autónoma Benito Juárez de Oaxaca.

Dr. Hugo Neftalí Padilla. Universidad Estatal de Sonora

Dra. María Teresa Gaxiola Sánchez. Universidad de Sonora.

Dr. José Cesar Kaplan. Universidad Estatal de Sonora.

Dr. Alfredo Islas Rodríguez. Universidad de Sonora

Frecuencia de publicación: semestral / 2 números por año.

Revista de Investigación Académica sin Frontera (RIASF) con (ISSN: 2007-8870) es un interlocutor internacional de acceso abierto revisado diario en línea en el ámbito del de las Ciencias Económicas Administrativas y Sociales. Su objetivo principal es dar a los trabajos de investigación de calidad. Cubre todas las sub-campos de los campos anteriormente mencionados. Proporciona la plataforma a académicos, estudiantes y profesionales. Sólo pública trabajos de investigación y artículos de revisión inicial. Documento presentado debe cumplir con algunos criterios como, debe ser original, inédita y no estén sometidos a ninguna otra revista.

RIASF es una revista arbitrada / Revisión por pares International. Publicamos documentos sobre una variedad de temas, contextos y estrategias de análisis que examinan la relación entre la rápida evolución para la Sociedad y la tecnología del conocimiento.

REVISTA DE INVESTIGACIÓN ACADÉMICA SIN FRONTERA, Año 13, No. 33, Julio - diciembre 2020, es una publicación semestral de investigación científica, editada por la Universidad de Sonora, a través de las División de Ciencias Económicas y Sociales, de la Unidad Regional Sur, Blvd. Lázaro Cárdenas No. 100, Col. Francisco Villa, Navojoa, Sonora, Sonora, México, C.P. 85880. Tel. (642) 425- 99-54.

http://www.revistainvestigacionacademicasinfrontera.com/, revistaacademicasinfrontera@ unison.mx. 


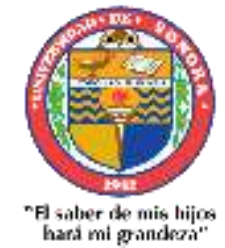

Año 13.

Núm. 33

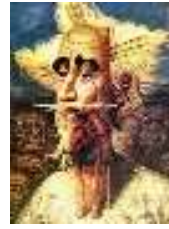

Revista de Investigación

Académica sin Frontera

ISSN: 2007-8870

\section{https://revistainvestigacionacademicasinfrontera.unison.mx/index.php/RDIASF}

Recibido el 27 de junio de 2020. Dictaminado mediante arbitraje favorablemente 7 de octubre de 2020 .

Editor responsable: Francisco Espinoza Morales. Reserva de Derechos al Uso Exclusivo: 04-2013121811323700-203 e ISSN: 2007-8870, ambos otorgados por el Instituto Nacional de Derecho de Autor. Inscrita en el Directorio de LATINDEX, con Núm. De folio 20014, folio único 14590. Responsable de la última actualización de este Número, Unidad Informática de la Universidad de Sonora, fecha de la última modificación, 30 de diciembre 2020, indexada a Cite Factor Academic Scientific Journal y Journals Master (IIJIF) y Red Latinoamericana de Revistas Académicas en Ciencias Sociales y Humanidades, (Latín Rev). Las opiniones expresadas por los autores no necesariamente reflejan la postura del editor de la publicación. Se autoriza la reproducción total o parcial de los contenidos e imágenes en la presente publicación siempre y cuando se cuente con la autorización del editor y se cite plenamente la fuente.

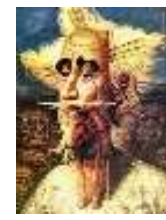

Nos complace anunciar que su diario, "Academic Research Journal Withoutborders" (ISSN/EISSN 2007-8870) fue evaluado positivamente en la indexación Citefactor, ahora la página de la revista está disponible en línea, en caso de cualquier problema.

$$
\text { Journals Master | International Innovative Journal Impact Factor (IIJIF) }
$$

Red Latinoamericana de revistas Académicas en Ciencias Sociales y Humanidades 
"Fl wither ide mis hijes
harfi mi prondersi"

Año 13.

Núm. 33

https://revistainvestigacionacademicasinfrontera.unison.mx/index.php/RDIASF
Revista de Investigación

Académica sin Frontera

ISSN: 2007-8870

Recibido el 27 de junio de 2020. Dictaminado mediante arbitraje favorablemente 7 de octubre de 2020 .

Impact Foctor (III))

https://www.neliti.com

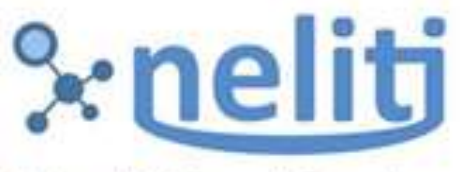

Indonesia's Research Repository

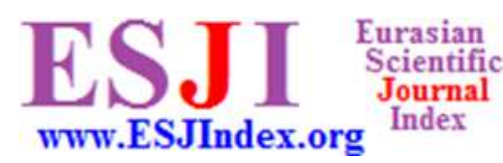

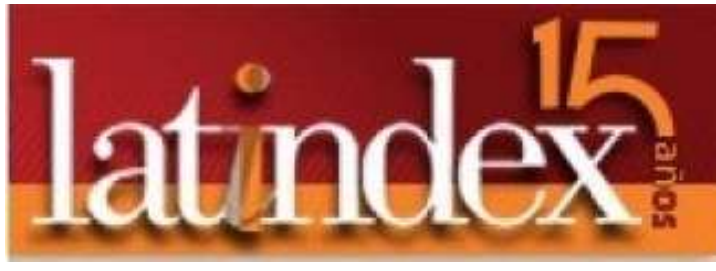

Academic

Resource

Index

ResearchBib 